\title{
Rosseland and Planck mean opacities for protoplanetary discs ${ }^{\star}$
}

\author{
D. Semenov ${ }^{1}$, Th. Henning ${ }^{2}$, Ch. Helling ${ }^{4}$, M. Ilgner ${ }^{3}$, and E. Sedlmayr ${ }^{4}$ \\ 1 Astrophysical Institute and University Observatory, Schillergäßchen 2-3, 07745 Jena, Germany \\ 2 Max-Planck-Institute for Astronomy, Königstuhl 17, 69117 Heidelberg, Germany \\ ${ }^{3}$ Institute of Astronomy and Astrophysics, Auf der Morgenstelle 10, 72076 Tübingen, Germany \\ 4 Center for Astronomy and Astrophysics, TU Berlin, Hardenbergstraße 36, 10623 Berlin, Germany
}

Received 1 April 2003 / Accepted 8 August 2003

\begin{abstract}
In this paper, we present mean gas and dust opacities relevant to the physical conditions typical of protoplanetary discs. As the principal absorber for temperatures below $\sim 1500 \mathrm{~K}$, we consider spherical and aggregate dust particles of various sizes, chemical structure, and porosity, consisting of ice, organics, troilite, silicates, and iron. For higher temperatures, ions, atoms, molecules, and electrons are included as the main opacity sources. Rosseland and Planck mean opacities are calculated for temperatures between $5 \mathrm{~K}$ and $10000 \mathrm{~K}$ and gas densities ranging from $10^{-18} \mathrm{~g} \mathrm{~cm}^{-3}$ to $10^{-7} \mathrm{~g} \mathrm{~cm}^{-3}$. The dependence on the adopted model of dust grains is investigated. We compare our results with recent opacity tables and show how different opacity models affect the calculated hydrodynamical structure of accretion discs.
\end{abstract}

Key words. accretion, accretion discs - hydrodynamics - atomic processes - molecular processes stars: planetary systems: protoplanetary discs

\section{Introduction}

Recently, significant progress toward the understanding of the possible composition and properties of dust grains and gas species in many astrophysical environments has been achieved. For instance, (sub)millimetre observations of molecular lines provided basic information about chemical composition and dynamical properties of the gas in discs around pre-mainsequence stars and young stellar objects (e.g., Bujarrabal et al. 1997; Olofsson et al. 2001; Thi et al. 2001; Piétu et al. 2003). The infrared-to-millimetre continuum observations of such environments constrain the properties of dust grains and can be used to estimate masses and thermal structure of the objects (e.g., Bouwman et al. 2000; Boogert et al. 2002; Tuthill et al. 2002). Finally, experimental studies on the formation and spectra of various gaseous species (e.g., Butler et al. 2001; Sanz et al. 2002) as well as the composition and properties of meteoritic, cometary, and interplanetary dust together with their laboratory analogues (e.g., Chihara et al. 2002; Mutschke et al. 2002) form a basis for theoretical investigations.

On the other hand, the increase of computer power and new numerical methods stimulate the development of more

Send offprint requests to: D. Semenov,

e-mail: dima@astro.uni-jena.de

* Appendix A is only available in electronic form at http://www. edpsciences.org sophisticated hydrodynamical models (e.g., Kley et al. 2001; D’Angelo et al. 2002; Struck et al. 2002). Many of such simulations need an accurate treatment of the energy transport within the dusty medium (see e.g., Klahr et al. 1999; Niccolini et al. 2003), which requires a detailed description of the radiative properties of matter. Consequently, the adopted opacity model is an important issue.

In this paper, we deal with physical conditions typical for protostellar nebulae and protoplanetary discs around low-mass young stellar objects. Virtually everywhere within the medium dust grains are the main opacity source, as they absorb radiation much more efficiently compared to the gas and because the temperature in these regions is low enough to prevent their destruction. However, for hotter domains ( $T \gtrsim 1500 \mathrm{~K})$, where even the most stable dust materials cannot survive, it is necessary to take absorption and scattering due to molecular species into account.

Recently, several extensive models describing the properties and evolution of dust grains in protostellar cores and protoplanetary discs were proposed by Henning \& Stognienko (1996), Schmitt et al. (1997), and Gail (2001, 2002).

Henning \& Stognienko (1996) studied the influence of particle aggregation on the dust opacity in the early evolutionary phases of protoplanetary discs. They concluded that distribution of iron within the particles affects their optical properties 
in a great respect. Schmitt et al. (1997) for the first time investigated collisional coagulation of dust grains in protostellar and protoplanetary accretion discs coupled with hydrodynamical evolution of these objects. They reported significant alteration of the thermal disc structure caused by the modification of the opacity due to dust growth. Gail $(2001,2002)$ considered annealing and combustion processes leading to the destruction of silicate and carbon dust grains consistently with the evolution of a steady-state accretion disc. They found that the modification of the dust composition in the inner regions due to these processes and its consequent transport toward outer disc domains affect the opacity and, eventually, the entire disc structure.

A number of papers deal with the computation of Rosseland or/and Planck mean gas opacities in atmospheres of cool stars, protostars, and stellar winds. Alexander \& Ferguson (1994) computed a set of opacity tables for temperatures between $700 \mathrm{~K}$ and $12500 \mathrm{~K}$ for several compositions. They considered a condensation sequence ${ }^{1}$ of refractory materials based on chemical equilibrium calculations and took into account absorption and scattering properties of these solids as well as various gas species. Note that the actual formation process is not modelled by such calculations. Helling et al. (2000) calculated gas mean opacities for wide ranges of density, temperature and various chemical compositions based on up-todate spectral line lists of the Copenhagen SCAN data base and studied the importance of the molecular opacity for the dynamics of the stellar winds of cool pulsating stars. An extension of this work will be used to construct our opacity model for protoplanetary accretion discs.

In Appendix A, we give a brief overview of the most common opacity models and studies and where they have been applied. It can be clearly seen that there is a lack of papers which focus on calculations of both Rosseland and Planck mean opacities of grain and gas species for temperatures between several $\mathrm{K}$ and few thousands $\mathrm{K}$ in a wide range of densities based on both the best estimates of the dust composition and properties and recent improvements in molecular line lists.

The first goal of this paper is to define such a model. In addition, we study how Rosseland and Planck mean opacities depend on the properties of dust grains and compare them with other opacity tables. Furthermore, we investigate how different opacity models affect the hydrodynamical structure of accretion discs. Our opacity model is freely available in the Internet: http://www . astro.uni-jena.de/Laboratory/ labindex.html

The paper is organised as follows. We introduce the opacity model in Sect. 2. The influence of the grain properties on resulting Rosseland mean opacities is described in Sect. 3.1. The Rosseland and Planck mean opacities are compared to other recent opacity tables in Sect. 3.2. We study how different opacity tables affect the hydrodynamical structure of active accretion discs in Sect. 3.3. A summary follows in Sect. 4. Finally, an overview of the opacity models cited in the paper is given in Appendix A.

\footnotetext{
1 Which is not to be confused with a formation sequence (see also Woitke 2000).
}

\section{The opacity model}

\subsection{Dust opacities}

In order to calculate frequency-dependent, Rosseland and Planck mean opacities of dust grains, we partly follow the schemes, proposed in Pollack et al. (1994, hereafter PHB), Henning \& Stognienko (1996, HS), and Voshchinnikov \& Mathis (1999, VM).

\subsubsection{Composition model and grain sizes}

In this paper we follow the dust composition model for accretion discs by Pollack et al. (1994; Sect. 2.3) which is based on an analysis of a wide range of theoretical, laboratory, and observational dust data. This composite model has been frequently used, for instance, in the evolutionary modelling of accretion discs around young stellar objects or estimates on their mass from millimetre observations (e.g., Greaves et al. 1998; Jura \& Werner 1999; D’Alessio et al. 2001).

The main dust constituents include amorphous pyroxene $\left([\mathrm{Fe}, \mathrm{Mg}] \mathrm{SiO}_{3}\right)$, olivine $\left([\mathrm{Fe}, \mathrm{Mg}]_{2} \mathrm{SiO}_{4}\right)$, volatile and refractory organics (CHON material), amorphous water ice, troilite $(\mathrm{FeS})$, and iron $^{2}$. Following HS, we vary the relative iron content in the silicates considering "iron-rich" (IRS) silicates with $\mathrm{Fe} /(\mathrm{Fe}+\mathrm{Mg})=0.4$, "normal" silicates $(\mathrm{NRM})$ with $\mathrm{Fe} /(\mathrm{Fe}+\mathrm{Mg})=0.3$, and "iron-poor" (IPS) silicates with $\mathrm{Fe} /(\mathrm{Fe}+\mathrm{Mg})=0$. However, the absolute amount of metallic iron in all these models is kept constant, which leads to the absence of solid iron in the first case and enhanced mass fraction of Fe in the third case. Such a variety of silicate models allows us to study the influence of iron content within the grain constituents on the extinction properties of dust. Another reason is that the exact mineralogical composition of the silicates in the protostellar clouds and protoplanetary discs is poorly constrained and can be different for various environments. Compared to HS, we re-estimated the absolute abundances of the silicates $\left(\mathrm{Fe}_{x} \mathrm{Mg}_{1-x} \mathrm{SiO}_{3}, \mathrm{Fe}_{2 x} \mathrm{Mg}_{2 x-2} \mathrm{SiO}_{4}\right)$, iron, and troilite $(\mathrm{FeS})$ in the cases of the "iron-poor" and "iron-rich" models from the Fe-stocheometric fractions keeping constant the total amount of iron. For the mass fractions of all dust constituents and their densities we follow Table 2 in PHB. Note the difference between the iron mass fractions in the different dust models.

The sublimation temperatures of the grain constituents are adopted from PHB (see Table 3 therein). We suppose that destruction of dust materials occurs in a narrow range of temperatures $(\sim 10-30 \mathrm{~K})$. Given that the evaporation of the silicates and iron happens at approximately the same conditions, we do not distinguish between their evaporation temperatures and assume that they evaporate in one wide temperature range, $\sim 100 \mathrm{~K}$. Thus, we account for six principal temperature regions:

1. $T \lessgtr 155 \mathrm{~K}$ - all dust constituents are present;

2. $\sim 165 \mathrm{~K}<T \lesssim 270 \mathrm{~K}-$ no ice;

\footnotetext{
${ }^{2}$ See: http://www.astro.uni-jena.de/Laboratory/ labindex.html
} 
3. $\sim 280 \mathrm{~K}<T \lesssim 410 \mathrm{~K}-$ no ice and volatile organics;

4. $\sim 440 \mathrm{~K}<T \lesssim 675 \mathrm{~K}$ - silicates, iron, and troilite are present;

5. $\sim 685 \mathrm{~K}<T \lesssim 1500 \mathrm{~K}-$ silicates and iron are present;

6. $\sim 1500 \mathrm{~K}<T \lesssim 10000 \mathrm{~K}$ - gas-dominated opacities.

We take into account the dependence of the evaporation temperatures of ice, silicates, and iron on gas density (the values shown above are given for a density $\sim 10^{-10} \mathrm{~g} \mathrm{~cm}^{-3}$ ). Note that if one considers a condensation sequence of these materials (hysteresis behaviour), it results in higher values of the condensation temperatures compared to the evaporation temperatures.

We assume that for the fifth temperature region the absolute amount of solid iron raises due to the destruction of troilite. The corresponding mass fractions of metallic iron are $6.15 \times 10^{-4}$, $2.42 \times 10^{-4}$, and $1.29 \times 10^{-3}$ for the NRM, IRS, and IPS silicate models, respectively.

As for the size distribution, we apply a modified MRN (Mathis et al. 1977) function suggested by Pollack et al. (1985). The modification consists of the inclusion of large $(0.5 \mu \mathrm{m}-$ $5 \mu \mathrm{m})$ dust grains. Such particle growth is expected to proceed efficiently at the early phases of the protoplanetary disc evolution due to the coagulation of small dust grains (see, for instance, Mizuno et al. 1988). We do not consider other size distributions since the overall effect of the particle sizes on the dust opacity is well studied (e.g., Pollack et al. 1985; Beckwith et al. 2000). It further allows us to compare directly our results with other works.

\subsubsection{Grain structure and topology}

It becomes evident from theoretical investigations and laboratory experiments that the dust agglomeration is an efficient process in dense and relatively cold environments, like protostellar cores or protoplanetary discs (e.g., Kesselman 1980; Nuth \& Berg 1994; Ossenkopf \& Henning 1994; Kempf et al. 1999; Wurm \& Blum 1998, 2000; Blum et al. 2002). Agglomeration leads to the formation of irregular particles consisting of hundreds or thousands of tiny subgrains. Usually, dust aggregates of two extreme kinds are considered, depending on the assumed coagulation processes, namely, PCA (particle-cluster aggregation) and CCA (cluster-cluster aggregation). As the laboratory and theoretical studies reveal, the PCA aggregates are sphere-like particles having a fractal dimension of about 3 . They have a compact "core" and a more rarefied "mantle". The CCA process results in the formation of very filamentary grains with complicated structure. They have fractal dimension of roughly 2 (Stognienko et al. 1995).

During the evolution of parent objects, like molecular clouds or protostellar discs, chemical and physical processes can further modify the properties of dust grains. For instance, accretion of volatile materials on dust surfaces and their subsequent chemical processing are efficient in outer regions of protoplanetary discs and in protostellar clouds (e.g. Greenberg 1967; Brown et al. 1988; Hartquist \& Williams 1990; Hasegawa \& Herbst 1993; Willacy et al. 1994; Aikawa et al. 1999). This results in well-defined "core-mantle" or, more probably, "onion-like" grain structure. In protostellar discs, dust can be transported by the accretion flow toward hotter regions, where their volatile mantle materials evaporate, and sputtering, annealing, combustion, and crystallisation processes may change the structure, composition, and sizes of the grains (Gail 2001, 2002). Therefore, it seems obvious that the real astronomical grains must have a very complicated structure and topology.

Unfortunately, modern computational methods and facilities allow only the consideration of somewhat simplified (but still reasonable) types of dust grains. In the present study, we focus on the following particle types:

1. homogeneous and composite aggregates;

2. homogeneous, composite, and porous composite spherical particles;

3. multishell and porous multishell spherical particles.

An aggregate dust particle is assumed to be a cluster of small spherical subgrains sticking together. A particle is called "homogeneous" if it consists of only one dust component. On the contrary, "composite" means that a particle incorporates a fine mixture of various materials (heterogeneous particle). In addition to these two extreme cases of the chemical dust structure, we consider a model of "multishell" grains, where each particle includes all constituents distributed within concentric spherical shells. To study the influence of the porosity on the extinction properties of dust grains, we fill composite and multishell spherical particles with vacuum. It is reasonable to assume that the optical behaviour of these porous multishell and porous composite particles may resemble that of more realistic kinds of dust grains.

\subsubsection{Computational methods}

The aggregate model and the numerical method to compute the optical properties of coagulated particles are adopted from HS. The aggregates are assumed to be in the form of PCA (50\%) and CCA (50\%) particles consisting of $0.01 \mu \mathrm{m}$ spherical subgrains. The spectral representation of inhomogeneous media (Bergman 1978) and the discrete multipole method (DMM) are elaborated to calculate effective dielectric functions of aggregates (Stognienko et al. 1995). At first, we use the DMM to calculate the spectral function of the aggregated particles of a special topology, when their subgrains touch each other only at one point. Then, we account for the interaction strength between the subgrains (percolation), which varies with the size and type of the aggregates, by an analytical expression (see Eq. (13) in HS). Finally, the effective dielectric functions of the aggregates are obtained by the spectral representation (HS, Eq. (5)). In the case of the composite aggregates, we compute the optical constants of the composite material by the Bruggeman effective medium theory (Bruggeman 1935), generalised to many components by Ossenkopf (1991). The optical properties of the dust aggregates are calculated with the usual Mie theory. It should be noted that this numerical approach is valid in the static limit only, which means that the scale of inhomogeneities within the particles must be small compared to the wavelength. Given the $0.01 \mu \mathrm{m}$ size of the subgrains and the shortest wavelength of $0.1 \mu \mathrm{m}$ we considered, this condition is fulfilled. 
Table 1. Mass fractions $f_{j}$ and densities $\rho_{j}$ of dust constituents.

\begin{tabular}{|c|c|c|c|}
\hline \multirow{2}{*}{$\begin{array}{l}\text { Material } \\
\text { (grain species } j \text { ) }\end{array}$} & \multirow{2}{*}{$\begin{array}{l}\mathrm{NRM} \\
f_{j}\end{array}$} & IRS & IPS \\
\hline & & $f_{j}$ & $f_{j}$ \\
\hline Olivine & $2.64 \times 10^{-3}\left(3.49 \mathrm{~g} \mathrm{~cm}^{-3}\right)$ & $3.84 \times 10^{-3}\left(3.59 \mathrm{~g} \mathrm{~cm}^{-3}\right)$ & $6.30 \times 10^{-4}\left(3.20 \mathrm{~g} \mathrm{~cm}^{-3}\right)$ \\
\hline Iron & $1.26 \times 10^{-4}\left(7.87 \mathrm{~g} \mathrm{~cm}^{-3}\right)$ & - & $7.97 \times 10^{-4}\left(7.87 \mathrm{~g} \mathrm{~cm}^{-3}\right)$ \\
\hline Pyroxene & $7.70 \times 10^{-4}\left(3.40 \mathrm{~g} \mathrm{~cm}^{-3}\right)$ & $4.44 \times 10^{-5}\left(3.42 \mathrm{~g} \mathrm{~cm}^{-3}\right)$ & $1.91^{-3}\left(3.20 \mathrm{~g} \mathrm{~cm}^{-3}\right)$ \\
\hline Troilite & $7.68 \times 10^{-4}\left(4.83 \mathrm{~g} \mathrm{~cm}^{-3}\right)$ & $3.80 \times 10^{-4}\left(4.83 \mathrm{~g} \mathrm{~cm}^{-3}\right)$ & $7.68 \times 10^{-4}\left(4.83 \mathrm{~g} \mathrm{~cm}^{-3}\right)$ \\
\hline \multicolumn{4}{|l|}{ Refractory } \\
\hline organics & $3.53 \times 10^{-3}\left(1.50 \mathrm{~g} \mathrm{~cm}^{-3}\right)$ & $3.53 \times 10^{-3}\left(1.50 \mathrm{~g} \mathrm{~cm}^{-3}\right)$ & $3.53 \times 10^{-3}\left(1.50 \mathrm{~g} \mathrm{~cm}^{-3}\right)$ \\
\hline \multicolumn{4}{|l|}{ Volatile } \\
\hline organics & $6.02 \times 10^{-4}\left(1.00 \mathrm{~g} \mathrm{~cm}^{-3}\right)$ & $6.02 \times 10^{-4}\left(1.00 \mathrm{~g} \mathrm{~cm}^{-3}\right)$ & $6.02 \times 10^{-4}\left(1.00 \mathrm{~g} \mathrm{~cm}^{-3}\right)$ \\
\hline Water ice & $5.55 \times 10^{-3}\left(0.92 \mathrm{~g} \mathrm{~cm}^{-3}\right)$ & $5.55 \times 10^{-3}\left(0.92 \mathrm{~g} \mathrm{~cm}^{-3}\right)$ & $5.55 \times 10^{-3}\left(0.92 \mathrm{~g} \mathrm{~cm}^{-3}\right)$ \\
\hline
\end{tabular}

We use the numerical approach of VM to model (porous) composite and (porous) multishell dust particles. In this method, the composite grains are represented as spheres with many concentric shells, where each shell includes several layers of randomly distributed dust materials. The multishell grains are modelled exactly as the composite ones but each shell includes only one layer of a dust constituent. Then a generalised multilayered Mie theory can be applied to calculate their optical properties. As it has been shown by VM, a convergence in the optical behaviour of the multishell particles is achieved if the number of shells exceeds 3 and dust materials within shells are randomly ordered. In our calculations, we found that this number must be at least 20 , because the highly absorbing material troilite is used, which induces interference within the shells and prevents fast convergence. Thus, in our case, a typical composite grain is modelled as a spherical particle with about hundred shells. On the contrary, a multishell grain is represented by a spherical particle with only a few shells.

We modify somewhat the dust model for the case of multishell and composite spherical particles. We mixed the silicates and iron into one material with the Bruggeman mixing rule. A similar mixture of silicates, sulphides, and metals (GEMS, Glass with Embedded Metals and Sulphur) is found to be a common component of interplanetary dust particles (Rietmeijer \& Nuth 2000). However, the main reason for this change is the convergence failure of the applied numerical method for the case of multishell grains with iron layers. This is due to numerical uncertainty which arises in the calculation procedure for the Mie scattering coefficients in the case of highly absorbing materials, like iron (for further explanation, see Gurvich et al. 2001).

We assume that each dust component has a total volume fraction in a particle according to its mass fraction and density, as specified in Table 1. For example, for the first temperature region and in the case of IPS silicate mineralogy, the mixture of iron and silicates occupies $8.9 \%$, troilite $-1.6 \%$, refractory organics $-23.4 \%$, volatile organics $-6 \%$, and water ice $-60 \%$ of the entire particle volume, respectively. These values are similar in the case of NRM and IRS models. Thus, if the temperature is low, organics and ice are the dominant components of the dust grains.

Unlike to the case of the composite particles, in the case of multishell spherical grains it is assumed that the distribution of dust materials is not random but follows their evaporation sequence. Thus, for the first temperature region in the protoplanetary disc, multishell spherical grains consist of a refractory core made of a mixture of silicates and iron and subsequent shells of troilite, refractory organics, volatile organics, and water ice. For higher temperature ranges, the number of shells is smaller since some materials are evaporated. In total, the number of shells in the case of multishell spherical particles varies from 2 to 5 . For the fifth temperature range $(T>700 \mathrm{~K})$, where all troilite is converted to solid iron, we let this iron form an additional layer on the grain surface. It is an extreme and probably physically unjustified case, but this allows us to study the influence of the formation of a highly absorbing surface layer on resulting opacities.

The porosity of particles is treated in a simple manner by the addition of $50 \%$ of vacuum (by volume) inside. For the case of the porous composite spheres, we consider vacuum to be one of the grain constituents, which forms additional "empty" layers. In the case of the multishell grain model, we mix each shell with vacuum in the same way which is applied to create the composite spherical particles. That is, we subdivide each individual shell in many layers and fill some of them with vacuum according to the requested porosity degree.

With the two computational approaches described above, we calculated the ensemble averaged absorption and scattering cross section as well as albedo and the mean cosine of the scattering angle for all kinds of dust grains. Applying Eqs. (1)(5) from Pollack et al. (1985; see Table 1 of the present paper), the dust monochromatic opacity and, consequently, the Rosseland and Planck mean opacities were obtained for temperatures below roughly $1500 \mathrm{~K}$ and a density range between $10^{-18} \mathrm{~g} \mathrm{~cm}^{-3}$ and $10^{-7} \mathrm{~g} \mathrm{~cm}^{-3}$. A convenient analytical representation of the Rosseland and Planck mean opacities for every temperature region is provided as a 5-order polynomial fit. This representation allows to calculate the Rosseland and Planck mean dust opacities accurately $(\sim 1 \%)$ and quickly for 
any given temperature and density values within the model applicability range, which is important for computationally extensive hydrodynamical simulations. The corresponding fit coefficients can be found in the code ${ }^{3}$.

\subsection{Gas opacity}

The opacity of the very inner part of the protoplanetary accretion disc is dominated by various gaseous species. Here, the temperature is too high for dust to be present.

Compared to the calculation of dust opacities, the calculation of accurate Rosseland and Planck mean gas opacities is more challenging due to the large variation in frequency, temperature, and density of the absorption coefficient of numerous molecules, atoms, and ions. In addition, the body of data to be handled easily amounts to several millions of absorption lines per molecule.

Missing data for absorption lines are critical for the calculation of Rosseland mean gas opacity since it is dominated by transparent spectral regions due to the harmonic nature of the averaging process. Therefore, each Rosseland mean is always only a lower limit of the correct value. The opposite is true for the case of the Planck mean opacity - missing data for weak lines or bands cause an overestimation of the strong lines. Therefore, a Planck mean is always an upper limit of the case of ideally complete data.

The dust opacity model for protoplanetary accretion discs outlined in the previous sections is supplied by a new table of gas opacities assembled on the basis of Helling (1999; Copenhagen SCAN data base) and Schnabel (2001; HITRAN data base). The gas opacity model is outlined in Helling et al. (2000) and only a short summary is given here. The Rosseland and the Planck mean opacities are calculated from opacity sampled line lists. The data for the line absorption coefficients used in Helling et al. (2000) (CO - Goorvitch \& Chackerian 1994; TiO - Jørgensen 1994; SiO - Langhoff \& Bauschilder 1993; $\mathrm{H}_{2} \mathrm{O}$ - Jørgensen \& Jensen 1993; CH Jørgensen et al. 1996; CN, $\mathrm{C}_{2}-$ Jørgensen \& Larsson 1990; $\mathrm{C}_{3}$ - Jørgensen 1989; HCN, $\mathrm{C}_{2} \mathrm{H}_{2}$ - Jørgensen 1990) were supplemented by data from the HITRAN 96 database $\left(\mathrm{CH}_{4}, \mathrm{NH}_{3}\right.$, $\mathrm{HNO}_{3}, \mathrm{H}_{2} \mathrm{CO}, \mathrm{CO}_{2}, \mathrm{~N}_{2} \mathrm{O}, \mathrm{O}_{3}, \mathrm{SO}_{2}, \mathrm{NO}_{2}, \mathrm{HO}_{2}, \mathrm{H}_{2}, \mathrm{O}_{2}, \mathrm{NO}$, $\mathrm{OH}, \mathrm{N}_{2}$ ). The opacity sampling of the latter was carried out by Schnabel (2001). The set of continuum opacities and scattering includes continuum absorption from H I (Karzas \& Latter 1961), H ${ }^{-}$(John 1988), H+H (Doyle 1968), H $\mathrm{H}_{2}^{-}$(Somerville 1964), $\mathrm{H}_{2}^{+}$(Mihalas 1965), $\mathrm{He}^{-}$(Carbon et al. 1969), He I, C I, MgI, Al I, Si I (all from Peach 1970) as well as Thompson scattering on free electrons and Rayleigh scattering. Collisioninduced absorption has been considered for $\mathrm{H}_{2}-\mathrm{H}_{2}$ and $\mathrm{H}_{2}-\mathrm{He}$ according to Borysow et al. (1997).

The number densities of the ions, atoms and molecules are computed from an updated chemical equilibrium routine, including 14 elements and 155 molecules based on the JANAF table (electronic version of Chase et al. 1985; for more detail see Helling et al. 2000). The element abundances are chosen

\footnotetext{
3 http://www . astro.uni-jena.de/Laboratory/ labindex.html
}

mainly according to Anders \& Grevesse (1989), but have been updated for several elements (see Helling et al. 2000).

Using this approach, the Rosseland and Planck mean gas opacities were computed for temperature ranges between $500 \mathrm{~K}$ and $10000 \mathrm{~K}$ and for gas densities between $\sim 10^{-18} \mathrm{~g} \mathrm{~cm}^{-3}$ and $\sim 10^{-7} \mathrm{~g} \mathrm{~cm}^{-3}$. In contrast to the dust opacity, no simplified analytical expression can be found for the gas mean opacities because of their sensitive dependence on temperature and density. Thus, we apply a second-order interpolation in order to calculate the gas opacities for any given temperature and density value from tabulated values.

\subsection{Opacity table}

In order to assemble the opacity table, we take into account either only dust opacity data for low temperatures, $T \lesssim 1500 \mathrm{~K}$, or only gas opacity data for higher temperatures. As has been shown by many authors (e.g., Lenzuni et al. 1995), it is an accurate approach because dust dominates the absorption properties of matter whenever it is present. It has been shown that already $30 \%$ of total $\mathrm{SiO}$ content in the solid phase is enough to exceed even the Planck mean gas opacity (Helling 1999). This fraction will decrease with increasing impurity of the grains. However, for the dust-to-gas transitional region ( $T$ from $\sim 1400 \mathrm{~K}$ to $1600 \mathrm{~K}$ ), where the last dust grain population evaporates, it is necessary to calculate the opacity of dust and gas simultaneously. In this narrow temperature range the resulting Rosseland and Planck mean opacities are certainly going down by few orders of magnitude, so one can apply a simple linear interpolation to estimate their values with a good accuracy. Still, we note that our table may give only approximate opacity data for that temperature range.

\section{Results}

In this section, we present the opacities of all types of dust grains and discuss the differences between them. Second, we compare the Rosseland and Planck mean opacities with other recently published opacity models. Finally, we study the influence of the adopted opacity model on the accretion disc structure.

\subsection{Opacity and the dust models}

The Rosseland mean opacities $\kappa_{\mathrm{R}}$ computed for all dust models are presented in Fig. 1. We compared two silicate models, namely, the IPS (left panel) and the IRS (right panel). Shown are the following dust models: multishell spheres, composite aggregates, composite spheres, homogeneous aggregates, homogeneous spheres, porous multishell grains, and porous composite spheres.

The most prominent trends in behaviour of the Rosseland mean dust opacities can be summarised as follows:

1. There is a significant difference in the calculated dust opacity values between the aggregates, (porous) composite and (porous) multishell spherical particles for the first $(T \lesssim$ $150 \mathrm{~K})$ and fifth $(T \gtrsim 700 \mathrm{~K})$ temperature regions; 

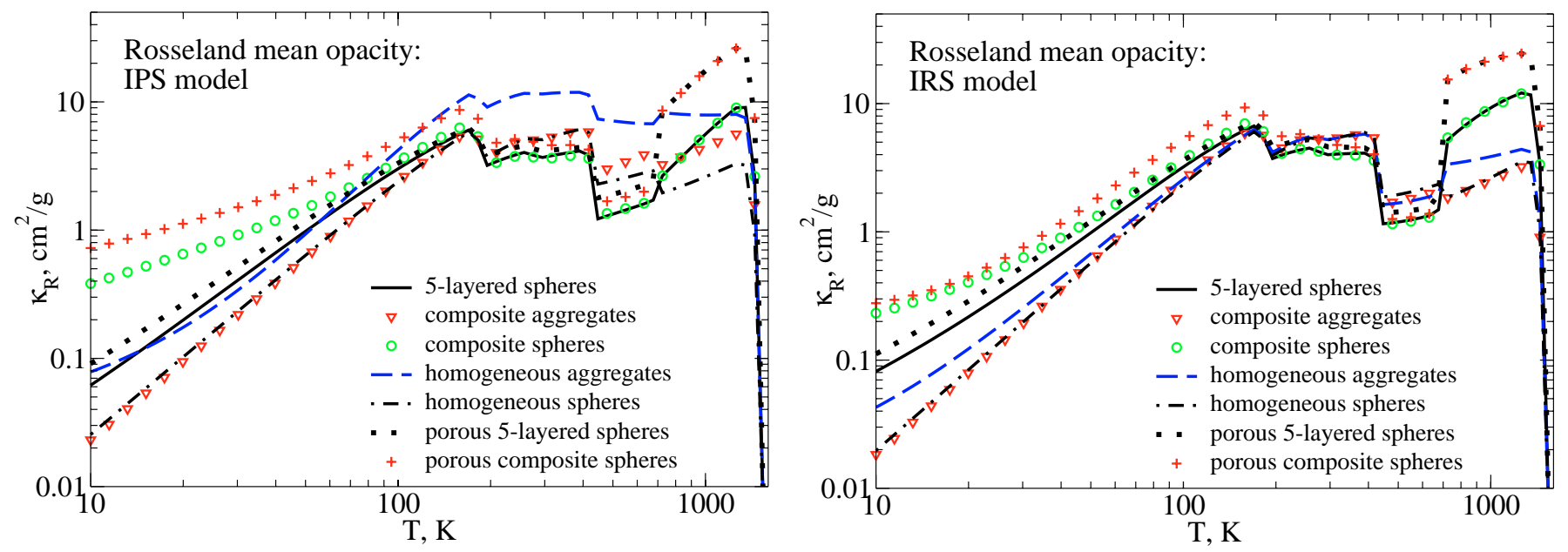

Fig. 1. The Rosseland mean opacities in the case of IPS (left panel) and IRS (right panel) silicate dust model. The following dust particle types are indicated: multishell spheres - solid line, composite aggregates - triangles, composite spheres - circles, homogeneous aggregates - dashed line, homogeneous spheres - dot-dashed line, porous multishell grains - dotted line, and porous composite spheres - pluses.

2. For intermediate temperatures, $150 \mathrm{~K} \lesssim T \lesssim 700 \mathrm{~K}$, the resulting Rosseland mean opacities do not show profound variations with the applied dust models, except for the case of the IPS homogeneous aggregates;

3. The discrepancy between opacity curves are smaller for the case of the IRS silicate model compared to the IPS;

4. Addition of vacuum inside compact composite and multishell spherical particles leads to significant modification of their opacities.

In the first temperature region, organics and water ice are the dominant dust materials according to our compositional model (see Table 1). At that temperatures $(T \lesssim 150 \mathrm{~K})$, the main contribution to the Rosseland mean opacity comes from monochromatic opacities in the wavelength range about $30 \mu \mathrm{m}-400 \mu \mathrm{m}$. In this spectral range, iron and troilite have higher values of the real $\left(n_{\lambda}\right)$ and imaginary $\left(k_{\lambda}\right)$ indices of refraction compared to the other materials considered in our model. For instance, at $\lambda=100 \mu \mathrm{m}$, iron has $n_{100 \mu \mathrm{m}}^{\mathrm{Fe}}=$ 95.02, $k_{100 \mu \mathrm{m}}^{\mathrm{Fe}}=181.95$, troilite has $n_{100 \mu \mathrm{m}}^{\mathrm{FeS}}=8.5, k_{100 \mu \mathrm{m}}^{\mathrm{FeS}}=$ 0.73 , whereas organics and water ice have $n_{100 \mu \mathrm{m}}^{\text {Org }}=2.14$, $k_{100 \mu \mathrm{m}}^{\text {Org }}=0.15$ and $n_{100 \mu \mathrm{m}}^{\text {Ice }}=1.82, k_{100 \mu \mathrm{m}}^{\text {Ice }}=0.05$, respectively. Furthermore, for troilite and especially for iron these values rapidly increase with wavelength. Thus, the resulting optical properties of a dust grain become sensitive to the absolute amount of $\mathrm{Fe}$ and $\mathrm{FeS}$ and its distribution inside the particle. In such a case, different theories to calculate optical properties of an aggregate particle may give different results and should be used with caution (see Stognienko et al. 1995, Figs. 6 and 8).

We considered aggregated particles of two kinds, namely, composite and homogeneous aggregates. The optical constants of the composite material do not show a peculiar behaviour at long wavelengths. Moreover, the refractive index of this composite changes only little if one is switching from the IPS to the IRS silicate model. One reason is that the amount of solid $\mathrm{Fe}$ is small compared to other constituent materials. Another reason is that the total iron abundance is kept constant in all compositional models. Hence the resulting optical properties of the composite aggregates are not very sensitive to the actual topology of the particles and adopted silicate model. Indeed, as it can be clearly seen in the figure, the dust opacity values of the composite aggregates (triangles) are close to the $\kappa_{\mathrm{R}}$ of the homogeneous spheres (dot-dashed line). The maximum deviation of these opacity curves is achieved in the case of the IPS silicates for temperatures higher than $\sim 700 \mathrm{~K}$. Here, the absolute amount of metallic iron is increased due to conversion of $\mathrm{FeS}$ to Fe. In addition, the Rosseland mean opacities of the composite aggregates do not depend much on the adopted silicate model (compare triangles on the left and right panel).

On the contrary, the $\kappa_{\mathrm{R}}$ values for the homogeneous aggregates do demonstrate a strong variations with the compositional model. In the case of the "iron-rich" silicates (right panel) the Rosseland mean values of the homogeneous aggregates (dashed line) lie much closer to the $\kappa_{\mathrm{R}}$ of the composite aggregates (triangles) then for the "iron-poor" compositional model (left panel). As it has been shown already by HS, this is caused by the presence of bare iron aggregates in the case of the IPS homogeneous aggregate model. Due to the extremely high absorptivity induced by the strong interactions between individual aggregate sub-grains, the optical properties of such iron clusters determine the overall behaviour of the resulting opacities. Note, that in the case of the "iron-rich" silicate composition, all iron is locked inside silicates and the absolute amount of troilite is reduced by a factor of two compared to the IPS and NRM models. In the absence of a population of highly absorbing grains, the Rosseland mean values of the homogeneous aggregates are close to that of the composite aggregates. However, at temperatures higher than $\sim 700 \mathrm{~K}$, troilite is converted to solid iron which increases the $\kappa_{\mathrm{R}}$ values of the homogeneous aggregates in respect to that of the composites (compare dashed lines with triangles on the right panel of Fig. 1).

The situation is different for the case of the (porous) composite and (porous) multishell spherical particles. As it was mentioned in Sect. 2.1.3, we changed the compositional model 

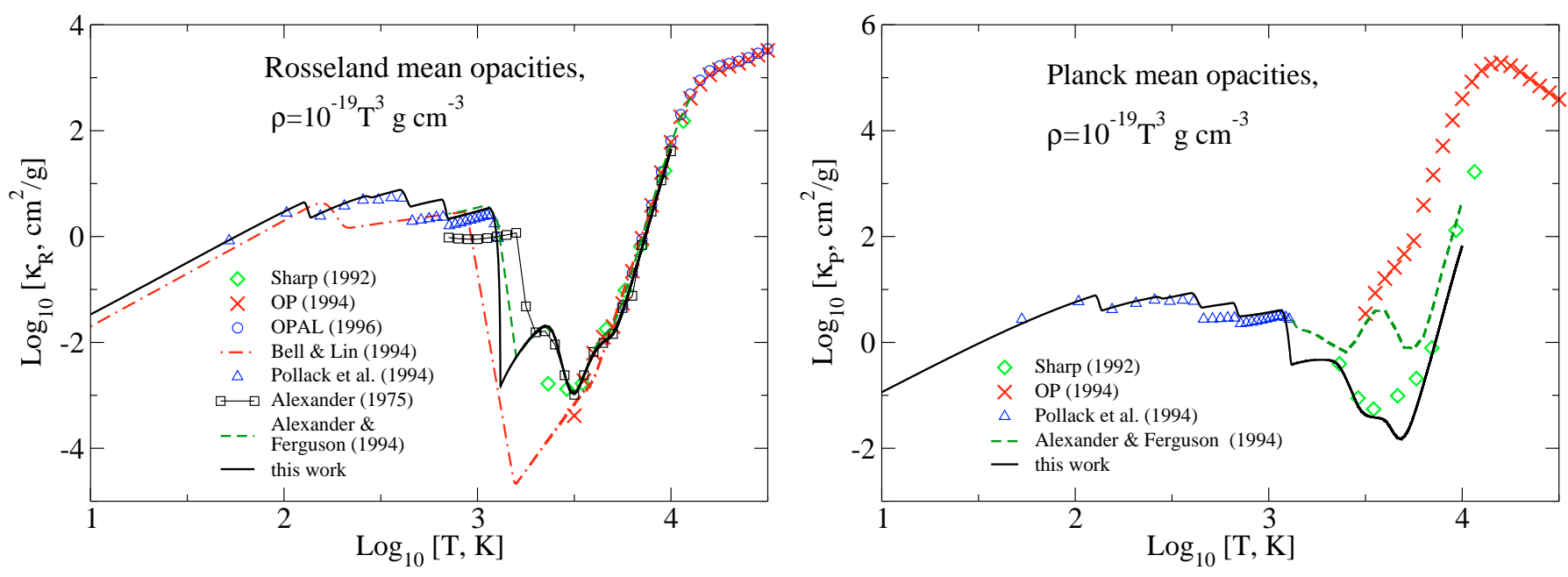

Fig. 2. The Rosseland (left panel) and Planck (right panel) mean opacities calculated for the whole temperature range considered ( $T \in$ $\left.\left[10,10^{5}\right] \mathrm{K}\right)$ and gas density scales as $10^{-19} \times T^{3} \mathrm{~g} \mathrm{~cm}^{-3}(\mathrm{C} / \mathrm{O}=0.43)$. Depicted are the mean opacities composed of the NRM composite aggregates in the low temperature range and the gas opacity for the higher temperatures (solid line). In comparison, opacity tables of the OP project (1994, crosses), OPAL project (1996, circles), Bell \& Lin (1994, dot-dashed line), Pollack et al. (1994, open triangles), Alexander (1975, line with open squares), Alexander \& Ferguson (1994, dashed line), and Sharp (1992, diamonds) are shown.

in this case and locked all solid iron in silicates using the Bruggeman rule of the effective medium theory. Thus, the only material with a high absorptivity at long wavelengths which remains in all compositional models is troilite. The metallic iron is another highly-absorbing dust component, but it is present in the fifth temperature region only, at $T>700 \mathrm{~K}$.

Voshchinnikov \& Mathis (1999) have shown that conventional EMTs are rather approximate in the case of small composite spherical particles, $x=2 \pi a / \lambda \ll 1$, when one of the grain constituents has a large refractive index (see Fig. $3 b$ therein). Given a typical size $a=0.1 \mu \mathrm{m}$ of our dust grains, a typical wavelength of $\lambda=100 \mu \mathrm{m}$ for the first temperature region, and the high refractive index of troilite at that wavelength, this condition is fulfilled. Thus, we adopted the approach of VM to model a composite grain as a spherical particle with many concentric layers and applied it for all temperature regions. Note that there is always an interference between the layers, which makes the optical properties of such composite particles to be different from that of composite grains with well mixed dust constituents. This is especially true if one of the dust components has a high absorptivity. In our compositional model for layered spherical particles, this is troilite in the first temperature region while in the fifth region it is iron. Therefore, we may expect to see the difference between the Rosseland mean opacity values of the composite spheres and composite aggregates, particularly for $T \lesssim 150 \mathrm{~K}$ and $T>700 \mathrm{~K}$.

As it can be seen in Fig. 1, indeed the $\kappa_{\mathrm{R}}$ values in the case of the composite spherical particles are much higher than for the composite aggregates. For instance, at $T=10 \mathrm{~K}$ this difference can reach factors of 10 and 20 for the IPS and IRS compositions, respectively (compare circles and triangles on the left and right panels). For higher temperatures, the Rosseland mean opacity curves of the composite spheres and aggregates are close to each other till $T \sim 700 \mathrm{~K}$ is reached. In this temperature region, we assumed that iron forms a layer on the surface of the composite spherical grains. Such a layer "screens out" all underlying materials and totally dominates the optical behaviour of the entire particle. Due to this fact, the dust opacity values of the composite spheres in the fifth temperature region is nearly the same for both the IPS and IRS compositions.

The multishell spherical particles have a restricted number of layers compared to the composite spheres, namely from 2 to 5 , depending on the temperature region. The troilite layer is assumed to be the first layer after the silicate core and thus troilite is "hidden" inside. It prevents a strong interference between the consequent particle shells as it is the case for the composite spheres. Then one may expect that the $\kappa_{\mathrm{R}}$ values of the multishell spheres should be lower than that of the composite spherical particles, especially for the IPS model. As it can be clearly seen in Fig. 1, this is true for $T \lesssim 150 \mathrm{~K}$, whereas for higher temperatures both opacity curves almost coincide (compare solid line with circles). Hence, the actual distribution of dust constituents within a multilayered spherical particle is not that important for the relevant Rosseland mean opacities at $T \lesssim 150 \mathrm{~K}$.

The addition of vacuum inside the compact composite and multishell spherical grains leads to a significant increase of the corresponding $\kappa_{\mathrm{R}}$ values for the first and fifth temperature regions (compare solid line with dotted line and circles with pluses). The first reason is that the density of the porous grains becomes lower than the density of the compact particles. Second, for the porous spheres the relevant extinction efficiencies are higher compared to that of the compact spherical particles if some of the dust constituents have a particularly high absorptivity, like troilite in the first and iron in the fifth temperature region, respectively. This is due to the coherence between the particle layers. The interference is more intense for the case of the composite sphere since it has more concentric layers and a nearly homogeneous distribution of the dust constituents from the centre to the surface compared to the 
multishell spherical particle. Note that in the fifth temperature region, both composite and multishell spheres have a similar chemical structure, namely, a silicate core covered by iron mantle. Therefore, it is naturally to expect that they have a similar behaviour of the resulting Rosseland mean opacities.

As it can be clearly seen in Fig. 1, the $\kappa_{\mathrm{R}}$ values of the porous composite spheres are higher than that of the compact composite spherical particles at $T<150 \mathrm{~K}$ and $T>700 \mathrm{~K}$ by a factor of 2 for both the IPS and IRS compositions (compare pluses and circles). For other temperatures, the corresponding Rosseland mean curves lie close to each other. The situation is similar for the case of the multishell and porous multishell spherical particles (compare solid line with dotted line). As we expected, in the fifth temperature region the Rosseland mean opacities for the case of the porous composite and porous multishell spheres have almost the same values.

\subsection{Comparison to other studies}

In Fig. 2, the Rosseland (left panel) and Planck (right panel) mean opacities composed of the NRM composite aggregate for the low temperature range and gas opacities for the high temperature range are compared with other models. We plotted these values for a wide temperature range, $T$ from $\sim 10 \mathrm{~K}$ to $10^{5} \mathrm{~K}$ and for gas densities which scale as $10^{-19} \times T^{3} \mathrm{~g} \mathrm{~cm}^{-3}$. It allows us to make a comparison between the models in a wide temperature as well as density range simultaneously.

As it is clearly seen in Fig. 2 (left panel), the discrepancy between the Rosseland mean values provided by various models is negligible at high temperatures $(T \gtrsim 3000 \mathrm{~K})$. On the contrary, the Planck mean opacity values differ by a few order of magnitude in this temperature range (compare curves on the right panel). The reason is that the Rosseland mean is much less sensitive to differences in the material data than the Planck mean due to the nature of the averaging process. However, the Planck mean heavily depends on the adopted values of the band and line strengths which vary for different line lists and on the adopted chemical equilibrium constants ${ }^{4}$

Therefore, the $\kappa_{\mathrm{P}}$ of the Opacity Project (OP, crosses) are much larger than all the other opacity models since it combines more atomic opacity sources (see e.g. Table 3.3 in Helling 1999). This model does not contain molecules for temperatures $1500 \mathrm{~K}-5000 \mathrm{~K}$, which become important absorbers in this temperature range. The difference between the Planck mean opacity values in the case of the AF model (dashed line) and our model (solid line) may be caused by different molecular line data and a different set of chemical species adopted. The same

\footnotetext{
${ }^{4}$ Note that the chemical equilibrium constants, $K_{p}$, used by different authors can cause differences in the resulting opacity values because it affects the number density of species. The same effect will be caused by the neglect of the metal ions in the chemical equilibrium calculations (see, e.g., Helling et al. 2000). It is, however, not very straightforward to decide which line list is the most correct (for a discussion see Jørgensen 2003). We nevertheless dare to demonstrate the difficulties arising from comparing Planck mean opacities calculated by different authors because it has - to our knowledge - not been pointed out clearly in the literature. Only comparisons of Rosseland mean opacities are presented, e.g. by Alexander \& Ferguson (1994).
}

is true for the $\kappa_{\mathrm{P}}$ values of Sharp (1992, diamonds), which lay somewhat in between the Planck mean opacities of our model (solid line) and the values of Alexander \& Ferguson (dashed line).

For temperatures lower than about $1500 \mathrm{~K}$, dust grains are the main opacity source. As it has been shown by Pollack et al. (1994), in this case the difference between the Rosseland and Planck mean opacities computed for the same model is small, $\sim 30 \%$ (see Fig. 4b therein). The reason is that both opacities are dominated by continuum absorption and scattering rather than absorption lines in this case. In what follows, we focus on the low-temperature Rosseland mean opacities only $(T \lesssim 3000 \mathrm{~K})$.

The Rosseland mean opacity values $\kappa_{\mathrm{R}}$ computed by the model of Bell \& Lin (1994, BL) strongly deviate from the $\kappa_{\mathrm{R}}$ calculated by other models. For example, this difference can reach a factor of hundred for $T \sim 1500 \mathrm{~K}-1800 \mathrm{~K}$ (compare dot-dashed and solid lines in Fig. 2). The reason is that the BL data are a modification of the old analytical Lin \& Papaloizou (1980) opacities, which are based on the opacity tables of Alexander (1975) and Cox \& Stewart (1970a,b) supplemented by the data from Alexander et al. (1989) for $T<3000 \mathrm{~K}$. The model of Bell \& Lin includes dust grains of two types, namely, homogeneous icy and metallic spherical grains. Since we used the more advanced dust compositional model of PHB and a different set of evaporation temperatures, this causes lower $\kappa_{\mathrm{R}}$ values in the case of the BL model compared to the other models for $T \lesssim 1500 \mathrm{~K}$. For higher temperatures, $\sim 1500 \mathrm{~K}-3000 \mathrm{~K}$, this deviation is huge, $\sim 100$ times. As it has been shown by AF, the reason is that Bell \& Lin truncated monochromatic opacities of water, which is one of the main absorbers at such temperatures, at a too short wavelength in their calculations. The missing opacity data affect the resulting Rosseland mean opacity. They do, so far, not draw any conclusions regarding Planck mean opacities.

In overall, the opacity curves of all other models do not show such a strong difference between each other (compare triangles, dashed line, solid line, and line with open squares). Our dust model differs from the model of Pollack et al. by taking into account an aggregate nature of cosmic dust grains and a new set of optical constants, but dust size distribution, composition, and evaporation temperatures are the same. However, the difference between the Rosseland mean opacity values of these two model can reach about a factor of two (see also Fig. 5a in Henning \& Stognienko 1996).

The $\kappa_{\mathrm{R}}$ values of the opacity model of Alexander (1975, line with open squares) is lower for the dust-dominated temperature region $(T \lesssim 1500 \mathrm{~K})$ and higher for the dust-to-gas transitional region ( $1500 \mathrm{~K} \lesssim T \lesssim 1700 \mathrm{~K}$ ) compared to our model (solid line) by factors of 5 and 100, respectively. The reason is, as mentioned above, that we used an approximation to compute $\kappa_{\mathrm{R}}$ in that temperature region, where the last dust grains get evaporated, which is not a very accurate approach. On the contrary, the opacity model of Alexander assumes the presence (but no evaporation!) of small $0.1 \mu \mathrm{m}$ spherical silicate grains in a rather approximate way, assuming that all dust is homogeneously condensed when the gas becomes saturated. Since here a phase-transition takes place, a supersaturated gas would 

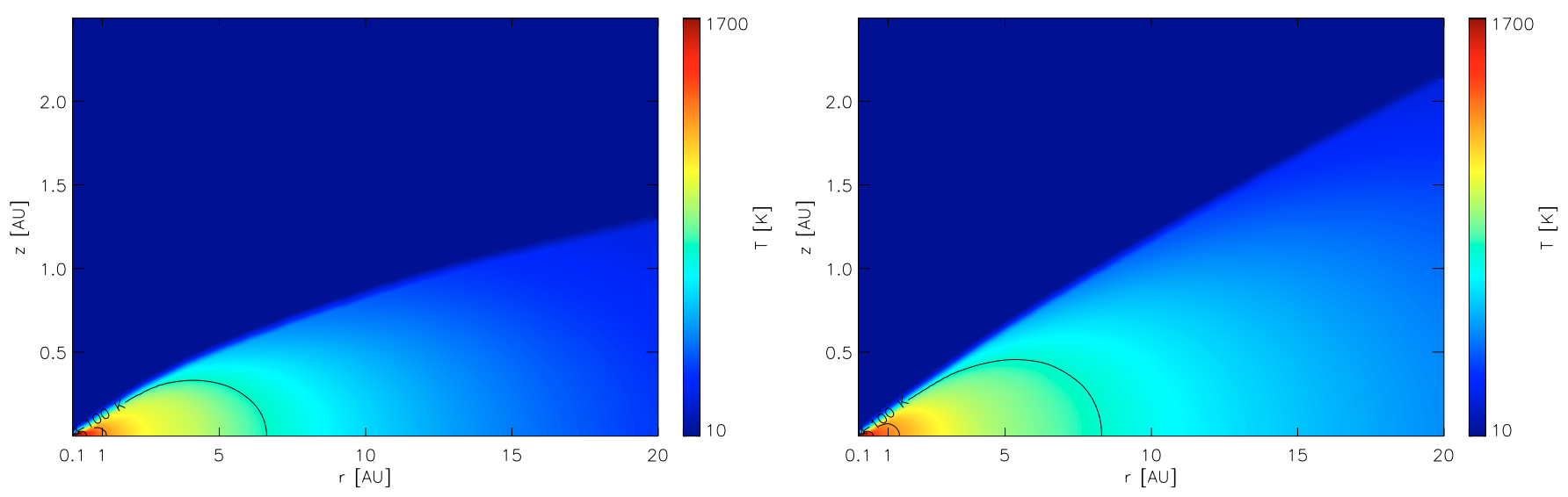

Fig. 3. The hydrodynamical structure of the accretion disc derived with the BL opacity model (left panel) and in the case of IPS homogeneous aggregates (right panel). The solid lines indicate temperatures of $1000 \mathrm{~K}, 500 \mathrm{~K}$, and $100 \mathrm{~K}$, respectively (from the left to the right side of the panels).

be needed which results in higher molecular abundances than AF derive from their equilibrium consideration (for a discussion see e.g. Woitke \& Helling 2003). Moreover, the condensation begins at higher temperature than the value we assumed for the evaporation of the last grain constituents because of the dust hysteresis. Finally, the model of AF neglects the presence of other refractory materials, like iron, in the dust-dominated temperature range, which makes the relevant Rosseland mean opacity values lower than provided by our model.

On the contrary, the model of AF does consider several dust materials, namely, iron, silicate, carbon, $\mathrm{SiC}$ assumed to be present as small ellipsoidal grain particles. Therefore, the corresponding Rosseland mean dust opacities nearly coincide with our values (compare dashed and solid lines, respectively). The same is true even for the dust-to-gas transitional region, where our opacity model gives rather approximate opacity values.

\subsection{Opacity and disc structure}

We compare the thermodynamical structure of a typical protoplanetary disc around a low-mass star computed with two different opacity tables in Fig. 3. The $1+1 \mathrm{D}$ model of an active steady-state accretion disc of Ilgner (2003) was used with the following input parameters: $M_{\star}=1 M_{\odot}, \dot{M}=10^{-7} M_{\odot} \mathrm{yr}^{-1}$, and $\alpha=0.01$. Here, $M_{\star}$ is the stellar mass, $\dot{M}$ is the mass accretion rate, and $\alpha$ is the parameter describing the kinematic viscosity. Note that this model incorporates a star as a gravitational center only and does not take into account the effect of the stellar radiation on the disc structure.

The thermal structure shown on the left panel was obtained with the Rosseland mean opacity table of Bell \& Lin (1994). On the right panel, we present the same disc structure but for the case of the IPS homogeneous aggregate model (IPSHA) supplemented by the gas mean opacity. We choose these two opacity models as the overall difference between them is the largest among the different models (compare solid and dashdotted lines in Fig. 2).
It can be clearly seen that the higher values of the Rosseland mean opacity in the case of the IPSHA model leads to a hotter and more extended disc structure. For instance, the vertical scale height of the disc at $20 \mathrm{AU}$ is equal to $1.3 \mathrm{AU}$ for the former and 2.1 AU for the latter opacity models, respectively. Consequently, there is also a variation of the density structure between the models, namely, the disc density is higher for the BL opacity model compared to that of the IPSHA model. The temperature difference is also prominent. For example, the midplane temperature of $100 \mathrm{~K}$, which roughly corresponds to the ice melting point, is reached at $6.5 \mathrm{AU}$ for the model of Bell \& Lin, whereas in the case of the IPSHA model it is at $\approx 8 \mathrm{AU}$.

To confirm our findings, we did a similar comparison with another code. We used a full 2D hydrodynamical code designed to simulate the interaction of the protoplanetary disc with a protoplanet (D'Angelo 2001). The parameters of the model were as follows: $M_{\star}=1 M_{\odot}, M_{\mathrm{d}}=0.01 M_{\odot}, v=10^{15} \mathrm{~cm}^{2} \mathrm{~s}^{-1}$, $\mu=2.39$, and $\gamma=1.4$, where $M_{\mathrm{d}}$ is the total disc mass, $v$ is the kinematic viscosity, $\mu$ is the mean atomic weight of the gas, and $\gamma$ is the adiabatic exponent.

The Bell \& Lin opacity model together with IPS and IRS homogeneous aggregate models were chosen for the comparison. The midplane temperature for all three opacity models is shown in Fig. 4. It can be clearly seen that the difference in the temperature values between all models can reach about $50 \%$ for the disc radii $r_{\mathrm{d}} \lesssim 2$ AU. Note that it disappears at larger distances, $r_{\mathrm{d}} \sim 10 \mathrm{AU}$. As expected, in the case of the BL model the midplane temperature is the lowest almost everywhere (dashed line), whereas for the IPS homogeneous aggregates it is the highest (solid line) and the IRS temperature values lie between them (dotted line). The reason is the same as for the case of the $1+1 \mathrm{D}$ disc model, namely, lower opacity values of the BL model compared to both the IPS and IRS opacities and higher values of IPS opacity in comparison with those of BL and IRS models. It is interesting that at $T \sim 130 \mathrm{~K}$ $(r \sim 2 \mathrm{AU})$ all the temperature curves are very close to each other. This is due to the adopted ice evaporation temperatures, which is a little lower in our case compared to the model of 


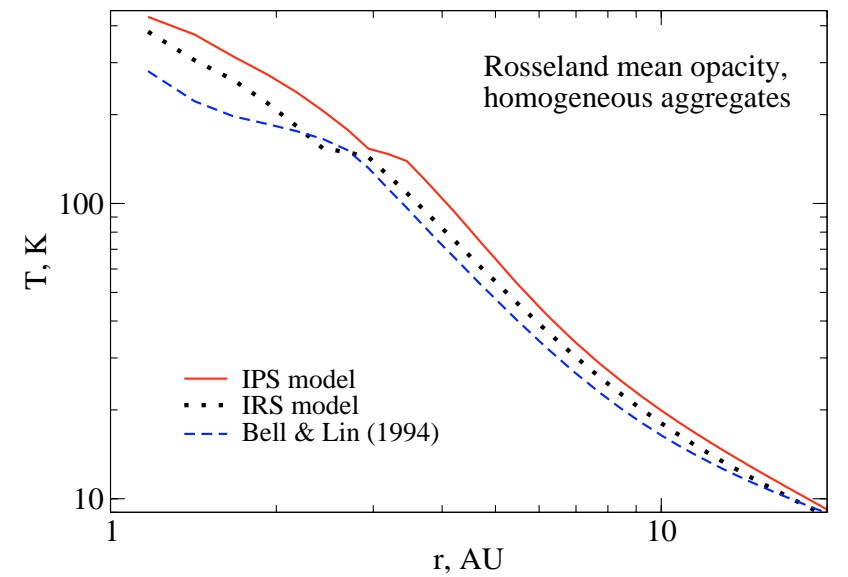

Fig. 4. The midplane temperature of the accretion disc obtained with the BL opacity model (dashed line) and IPS (solid line) and IRS (dotted line) homogeneous aggregate dust models.

Bell \& Lin. That leads to nearly the same opacity values for all three models at a restricted set of temperatures around $\sim 130 \mathrm{~K}$ (compare solid and dot-dashed lines in Fig. 3). For $r \gtrsim 10 \mathrm{AU}$ the temperature curves are close to each other because the corresponding opacity models have rather similar low-temperature opacity values.

Thus, we showed that the difference in the Rosseland opacity tables applied in hydrodynamical calculations leads to deviations in the resulting disc structure. As it has been shown by Markwick et al. (2002), the temperature distribution is a crucial factor for the chemical evolution in the inner parts of accretion discs. Therefore, proper opacity modelling is an important issue in order to follow the dynamical and especially chemical evolution of protoplanetary accretion discs.

\section{Summary}

We have compiled the Rosseland and Planck mean dust and gas opacities for the temperature range $T \in\left[5,10^{4}\right] \mathrm{K}$ and for gas densities $\rho \in\left[10^{-18}, 10^{-7}\right] \mathrm{g} \mathrm{cm}^{-3}$ which is appropriate for the conditions in protoplanetary discs. The absorption and scattering due to dust grains of different compositions, shapes and topological structures and the absorption provided by over 30 atoms, molecules, ions were taken into consideration. The corresponding well-documented numerical code together with representative data and figures are electronically available: http://www .astro.uni-jena.de/Laboratory/ labindex.html.

It has been found that the topological distribution of highly absorbing materials, such as iron and troilite, within dust grains dominates the relevant optical properties to a large degree. Particularly, the composite and porous composite spherical grains modelled in a special way have remarkably high Rosseland mean opacities at $T \lesssim 150 \mathrm{~K}$ and $T \gtrsim 700 \mathrm{~K}$ even in comparison with the composite aggregated particles. We have shown that at intermediate temperatures the Rosseland mean opacities of distinct grain models are close to each other. It has been demonstrated that the difference between the opacity values of various dust models is smaller in the case of the compositional model with a smaller amount of solid iron and troilite. We found that porous composite and porous multishell spherical particles show higher opacity values in comparison with their compact analogues.

We performed a comprehensive comparison of our results with other recent opacity models. We found a significant difference between the opacity models in the case of the Planck mean and a good agreement between them for the case of the Rosseland mean at $T \gtrsim 1500 \mathrm{~K}$, where gas species are the main opacity sources. For lower temperatures, where opacities are dominated by dust grains, there is a discrepancy (a factor of $\sim 3$ at most) in both the Rosseland and Planck mean values for all considered models.

We demonstrated that differences in the Rosseland mean opacity values provided by distinct opacity models affect the hydrodynamical structure of active steady-state accretion discs. Namely, higher values of the Rosseland mean opacity lead to a hotter and more extended disc structure in the case of $1+1 \mathrm{D}$ and 2D disc modelling.

Acknowledgements. DS was supported by the German Deutsche Forschungsgemeinschaft, DFG project "Research Group Laboratory Astrophysics" (He 1935/17-1). The work of MI was supported by the Max Planck Society. We are grateful to D. Alexander for valuable comments and discussions. For the calculations of the optical properties of multishell spherical particles, we used a code by N. Voshchinnikov (http://www.astro.spbu.ru/staff/ilin2/ ilin.html).

\section{References}

Aikawa, J., Umebayashi, T., Nakano, T., \& Miyama, Sh. 1999, ApJ, 519,705

Aikawa, T. 2001, A\&A, 371, 667

Aikawa, T., \& Antonello, E. 2000a, A\&A, 363, 593

Aikawa, T., \& Antonello, E. 2000b, A\&A, 363, 601

Alexander, D. R. 1975, ApJS, 29, 363

Alexander, D. R., \& Ferguson, J. W. 1994, ApJ, 437, 879

Alexander, D. R., Johnson, H. R., \& Rypma, R. L. 1983, ApJ, 272, 773

Alexander, D. R., Augason, G. C., \& Johnson, H. R. 1989, ApJ, 345, 1014

Anders, E., \& Grevesse, N. 1989, Geochim. Cosmochim. Acta, 53, 19

Beckwith, S., Henning, Th., \& Nakagawa, Y. 2000, in Protostars and Planets IV, ed. V. Mannings, A. P. Boss, \& S. S. Russell, 533

Bell, K. R., Cassen, P. M., Klahr. H. H., \& Henning, Th. 1997, ApJ, 486, 372

Bell, K. R., \& Lin, D. N. C. 1994, ApJ, 427, 987

Bergman, D. 1978, Phys. Rep., 43, 377

Blum, J., Wurm, G., Poppe, T., et al. 2002, AdSpR, 29, 497

Bodenheimer, P., Yorke, H. W., Rozyczka, M., \& Tohline, J. E. 1990, ApJ, 355, 651

Boogert, A., Hogerheijde, M., \& Blake, G. 2002, ApJ, 568, 761

Borysow, A., Jørgensen, U. G., \& Zheng, C. 1997, A\&A, 324, 185

Boss, A. 1988, ApJ, 331, 370

Bouwman, J., Meeus, G., Hony, S., et al. 2000, Planetary Systems in the Universe, IAU Symp., 202, 88

Brown, P., Charnley, S., \& Millar, T. 1988, MNRAS, 231, 409

Bruggeman, D. 1935, Ann. Phys., 24, 636 
Bujarrabal, V., Alcolea, J., Neri, R., \& Grewing, M. 1997, A\&A, 320, 540

Butler, R., De Lucia, F., Petkie, D., et al. 2001, ApJS, 134, 319

Carbon, D., Gingerich, O., \& Latham, D. 1969, Low Luminosity Stars, ed. S. Kumar (New York: Gordon and Breach)

Chase Jr., M. W., Davies, C. A., Downey Jr., J. R., et al. 1985, in J. Phys. Chem. Ref. Dat., Vol. 14, Suppl. 1 (National Bureau of Standards)

Chihara, H., Koike, C., Tsuchiyama, A., et al. 2002, A\&A, 391, 267

Collins, T. J. B., Helfer, H. L., \& Van Horn, H. M. 1998, ApJ, 502, 730

Cox, A. N., \& Stewart, J. N. 1970a, ApJS, 19, 243

Cox, A. N., \& Stewart, J. N. 1970b, ApJS, 19, 261

D’Alessio, P., Calvet, N., \& Hartmann, L. 2001, ApJ, 553, 321

D'Angelo, G. 2001, private communication

D’Angelo, G., Henning, Th., \& Kley, W. 2002, A\&A, 385, 647

Dalgarno, A., The Scattering of light by Atomic Systems. Volume III of Spectral Reflectivity of the Earth Atmosphere

Doyle, R. 1968, Ph.D. Thesis, Harvard University, Harvard, USA

Driebe, T., Blöcker, T., Schönberner, D., \& Herwig, F. 1999, A\&A, 350,89

Finocchi, F., Gail, H.-P., \& Duschl, W. J. 1997, A\&A, 325, 1264

Gail, H.-P. 2001, A\&A, 378, 192

Gail, H.-P. 2002, A\&A, 390, 253

Goorvitch, D., \& Chackeria Jr., C. 1994, ApJS, 91, 483

Greaves, J., Holland, W., Moriarty-Schieven, G., et al. 1998, ApJ, 506, L133

Greenberg, J. 1967, AJ, 72, 800

Grevesse, N., Lambert, D., Sauval, A., et al. 1991, A\&A, 242, 488

Gurvich, I., Shiloah, N., \& Kleiman, M. 2001, J. Quant. Spec. Radiat. Transf., 70, 433

Hartquist, T., \& Williams, D. 1990, MNRAS, 247, 343

Hasegawa, T., \& Herbst, E. 1993, MNRAS, 263, 589

Helling, Ch. 1999, Ph.D. Thesis, Technische Universität Berlin, Berlin, Germany

Helling, Ch. Winters, J. M., \& Sedlmayr, E. 2000, A\&A, 358, 651

Henning, Th., \& Stognienko, R. 1996, A\&A, 311, 291

Huré, J.-M. 2000, A\&A, 358, 378

Iglesias, C. A., \& Rogers, F. J. 1996, ApJ, 464, 943

Ikoma, M., Emori, H., \& Nakazawa, K. 2001, ApJ, 553, 999

Ilgner, M. 2003, Ph.D. thesis, Friedrich Schiller University, Jena, Germany

John, T. 1988, A\&A, 193, 189

Jørgensen, U. 1989, ApJ, 344, 901

Jørgensen, U. 1990, A\&A, 232, 420

Jørgensen, U. 1994, A\&A, 284, 179

Jørgensen, U. 2003, ASP Conf. Ser., 288, 303

Jørgensen, U., \& Jensen, P. 1993, J. Mol. Spect., 161, 219

Jørgensen, U., \& Larsson, M. 1990, A\&A, 238, 424

Jørgensen, U., Larsson, M., Iwamae, A., \& Yu, B. 1996, A\&A, 315, 204

Jura, M., \& Werner, M. 1999, ApJ, 525, L113

Karzas, W., \& Latter, R. 1961, ApJS, 6, 167
Kempf, S., Pfalzner, S., \& Henning, Th. 1999, Icarus, 141, 388

Kesselman, V. 1980, AZh, 57, 566

Klahr, H., Henning, Th., \& Kley, W. 1999, ApJ, 514, 325

Kley, W., D’Angelo, G., \& Henning, Th. 2001, ApJ, 547, 457

Langhoff, S., \& Bauschlicher Jr., C. 1993, Chem. Phys. Lett., 211, 305

Lenzuni, P., Gail, H.-P., \& Henning, Th. 1995, ApJ, 447, 848

Lin, D. N. C., \& Papaloizou, J. 1980, MNRAS, 191, 37

Lunine, J. I., Hubbard, W. B., Burrows, A., et al. 1989, ApJ, 338, 314

Markwick, A., Ilgner, M., Millar, T., \& Henning, Th. 2002, A\&A, 385, 632

Mathis, J. S., Rumpl, W., \& Nordsieck, K. H. 1977, ApJ, 217, 425

Mihalas, D. 1965, ApJS, 9, 321

Mizuno, H., Markiewicz, W., \& Völk, H. 1988, A\&A, 195, 183

Mutschke, H., Posch, Th., Fabian, D., \& Dorschner, J. 2002, A\&A, 392, 1047

Niccolini, G., Woitke, P., \& Lopez, B. 2003, A\&A, 399, 703

Nuth, J. III, \& Berg, O. 1994, In Lunar and Planetary Inst., The Twenty-Fifth Lunar and Planetary Science Conf. Part 2, 1011

Olofsson, G., Liseau, R., \& Brandeker, A. 2001, ApJ, 563, L77

Ossenkopf, V. 1991, A\&A, 251, 210

Ossenkopf, V., \& Henning, Th. 1994, A\&A, 291, 943

Peach, G. 1970, MmRAS, 73, 1

Peytremann, E. 1974, Astr. Ap., 33, 203

Piétu, V., Dutrey, A., \& Kahane, C. 2003, A\&A, 398, 565

Pollack, J. B., Hollenbach, D., Beckwith, S., et al. 1994, ApJ, 421, 615

Pollack, J. B., McKay, C. P., \& Christofferson, B. M. 1985, Icarus, 64, 471

Rietmeijer, F., \& Nuth III, J. 2000, Earth, Moon, and Planets, 82-83, 325

Rogers, F., \& Iglesias, C. 1992, ApJS, 79, 507

Ruden, S. P., \& Pollack, J. B. 1991, ApJ, 375, 740

Sacmann, I., Boothroyd, A. I., \& Kraemer, K. 1993, ApJ, 418, 457

Sanz, M., McCarthy, M., \& Thaddeus, P. 2002, ApJ, 577, L71

Schmitt, W., Henning, Th., \& Mucha, R. 1997, A\&A, 325, 569

Schnabel, K. 2001, Master Thesis, Technische Universität Berlin, Berlin, Germany

Seaton, M. J., Yan, Y., Mihalas, D., \& Pradhan, A. K. 1994, MNRAS, 266, 805

Sharp, C. M. 1992, A\&AS, 94, 1

Somerville, W. 1964, ApJ, 139, 192

Stognienko, R., Henning, Th., \& Ossenkopf, V. 1995, A\&A, 296, 797

Struck, C., Cohanim, B., \& Willson, L. 2002, ApJ, 572, 83

Thi, W., van Dishoeck, E., Blake, G., et al. 2001, ApJ, 561, 1074

Turcotte, S., Richer, J., Michaud, G., et al. 1998, ApJ, 504, 539

Tuthill, P., Monnier, J., Danchi, W., et al. 2002, ApJ, 577, 826

Voshchinnikov, N., \& Mathis, J. 1999, ApJ, 526, 257

Willacy, K., Rawlings, J., \& Williams, D. 1994, MNRAS, 269, 921

Woitke, P., in Astronomy with Radioactivities, ed. R. Diehl, \& D. Hartmann (Ringberg, Germany), MPE Rep., 274, 163

Woitke, P., \& Helling, Ch. 2003, A\&A, submitted

Wood, P. R. 1976, MNRAS, 174, 531

Wurm, G., \& Blum, J. 1998, Icarus, 132, 125

Wurm, G., \& Blum, J. 2000, ApJ, 529, 57 
D. Semenov et al.: Opacities for protoplanetary discs, Online Material $p 1$

\section{Online Material}




\section{Appendix A: An overview of the opacity models}

The goal of this compilation is to provide essential information about opacity models cited in the text. We show for which elemental composition they are developed, what kind of opacities they supply, and in what temperature and density ranges they work. In addition, key references to the studies, where these opacity models have been applied, are given and the aims of the investigations are briefly mentioned.

Primarily, we distinguish between two kinds of opacity models. The first kind is designed for stellar evolutions, where it is more convenient to use a special parameter, $R=\rho / T_{6}^{3}, T_{6}=T / 10^{6} \mathrm{~K}$ instead of gas density $\rho$ (see discussion in Rogers \& Iglesias 1992). Thus, opacity data of such models are assembled in rectangular tables in $R-T_{6}$ space. However, in hydrodynamical simulations of accretion discs, it is more convenient to have opacity tables composed in $\rho-T$ parameter space, for which we refer as to the second type of the models. The direct conversion of $R$ to $\rho$ in the opacity models of the first kind leads to trapezoidal tables, where opacity values for different temperatures have different density intervals. We mark such models with "a" and show the maximum limits of density and temperature values for them.

In addition, we point out if opacity data are available online in the Internet or via E-mail ("b"). By default, the papers are supposed to contain the opacity data in a tabular form. Otherwise, when only opacity plots are available, we label the corresponding papers with "c". The analytical opacity models are marked with "d". 


\section{Semenov et al.: Opacities for protoplanetary discs, Online Material p 3}

Table A.1. Opacity models cited in the text.

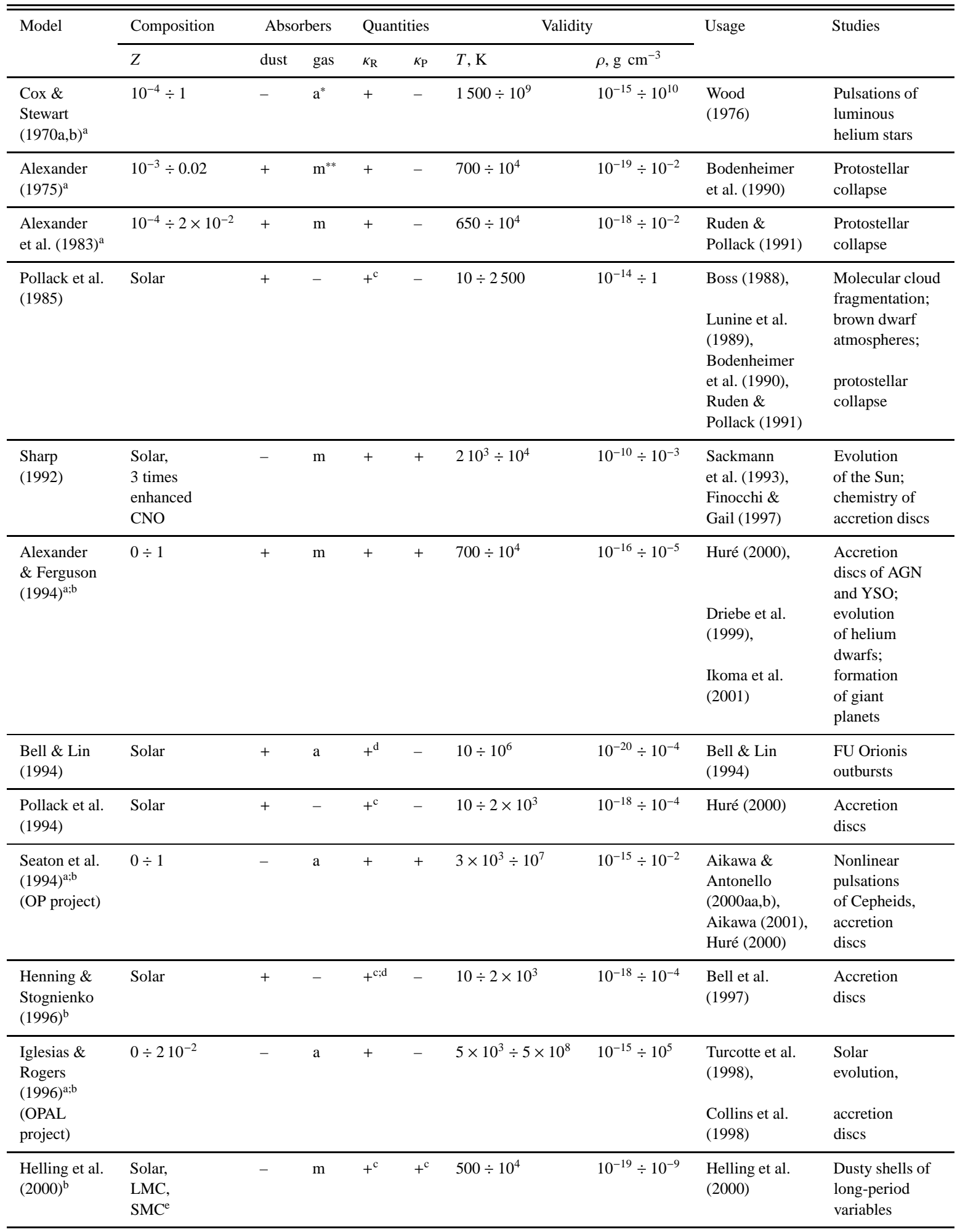

* Atomic opacities.

** Molecular and atomic opacities.

a A trapezoidal table in $\rho-T$ parameter space.

b It can be retrieved via E-mail or the Internet.

c Only plots are available.

d Analytical expressions are used.

e LMC, SMC mean the element abundances of the Large and the Small Magellanic Clouds. 